\section{E-088 TIMING AND OUTCOME OF ENDOVASCULAR THROMBECTOMY FOR IN-HOSPITAL STROKE: A SYSTEMATIC REVIEW AND META-ANALYSIS}

${ }^{1} S$ Almutairi, 'H Choudhury, ${ }^{2} \mathrm{M}$ Almekhlafi*. 'University of Calgary, Calgary, AB, CANADA; ${ }^{2}$ Clinical Neurosciences, Radiology, and Community Health Sciences, University of Calgary, Calgary, AB, CANADA

\subsection{6/neurintsurg-2020-SNIS.121}

Background and Purpose Acute strokes due to large vessel occlusion in hospitalized patients is not uncommon. Evidence is limited on the management and outlook of such patients. treatment in these patients. We performed a systematic review and meta-analysis to investigate the benefit and timing of endovascular thrombectomy (EVT) for in-hospital stroke.

Methods We conducted a systematic review and meta-analysis of clinical studies of ten patients or more published in English language until March 2020 in the MEDLINE and Cochrane databases. Searches were supplemented by scanning bibliographies of key articles. We included retrospective and prospective studies published in full and reported original data on the characteristics and outcomes of in-hospital stroke patients treated with EVT. Case reports or studies reporting the outcomes of mixed cohorts of intravenous thrombolysis or EVT were excluded if the characteristics of the EVT cohort were not reported separately. Two authors independently selected eligible studies and extracted data.

Results Out of 4996 retrieved studies, six studies were included with a total of 111 patients. The median age was 73.7 years (range 58-81 years) and median NIHSS was 17 (range 13-21). Patients were commonly admitted to the cardiology service $(24.3 \%)$ followed by surgery $(17.1 \%)$ and hematology (11.7\%). The most common stroke etiology was cardioembolic (61.3\%) and large artery disease (9\%), while $5.4 \%$ were malignancy-related. The most common occlusion sites were the middle cerebral (56\%) and terminal carotid (28\%) arteries. $21.6 \%$ received IV tPA. The pooled time from detection/last seen well to puncture was 134 minutes (95\% CI: 100.9 to 167.2 minutes) (figure A). Successful reperfusion was described in $81.1 . \%$ with a pooled detection to reperfusion time of 191.8 minutes (95\% CI: 167.6 to 216.1 minutes). Symptomatic intracranial hemorrhages occurred in $6.3 \%$. The cumulative probability of a good clinical outcome (mRS <3) was 39\% (95\% CI: 27 to 51\%) (figure B) with a cumulative mortality risk of 19\% (95\% CI: 11 to $27 \%$ )

Conclusions Despite hospitalization and existing co-morbidities, EVT safety and outcomes in the in-hospital stroke patients with large vessel occlusion are not different from communityonset cases. Noticeable delays from detection to puncture time are reported in the literature which call for better stroke pathways to identify and treat these patients fast.

Disclosures S. Almutairi: None. H. Choudhury: None. M. Almekhlafi: None.

\section{E-089 OUTCOMES OF MECHANICAL THROMBECTOMY IN PATIENTS WITH NEUROLOGICAL DISORDERS}

L Brasiliense*, H Abdulrazek, P Aguilar-Salinas, H Abbad, T Dumont, M El-Ghanem. Neurosurgery, University of Arizona, Tucson, AZ

\subsection{6/neurintsurg-2020-SNIS.122}

Introduction As the number of patients undergoing mechanical thrombectomy (MT) increases in the United States, operators may be confronted with patients harboring preexistent debilitating neurological disorders. Still, the epidemiology and reperfusion strategies of patients with acute ischemic stroke (AIS) and neurological disorders has not been established.

Methods The National Inpatient Sample (NIS) Database was queried for the most recent 5-year period available (20122016). We identified patients with a diagnosis of ischemic stroke and the following neurological disorders: Alzheimer's Disease (AD), Parkinson Disease (PD), Amyotrophic Lateral Sclerosis (ALS), Multiple Sclerosis (MS), and Myasthenia Gravis (MG). Variables analyzed included mechanical thrombectomy, discharge home, length of hospital stay (LOS), and inpatient mortality.

Results A total of 460,070 patients with ischemic stroke were identified. The prevalence of $\mathrm{AD}$ in this population was $2.3 \%$ $(10,775 / 460,070)$, PD was $1.2 \%(5,630 / 460,070)$, MS was $0.31 \%(1,430 / 460,070)$, ALS was $0.012 \%(56 / 460,070)$, and MG was $0.001 \%(9 / 460,070)$. Mechanical thrombectomy was performed in $1.63 \%$ of the patients $(7,529 / 460,070)$. Patients with co-existing neurological disorders underwent MT less often $(0.65 \% ; 118 / 17,900)$ compared to other patients $(1.67 \% ; 7,411 / 442,170)$. Patients with AD were significantly less likely to be discharged home after MT compared to patients without co-existing neurological disorders $(9 \%$ vs.
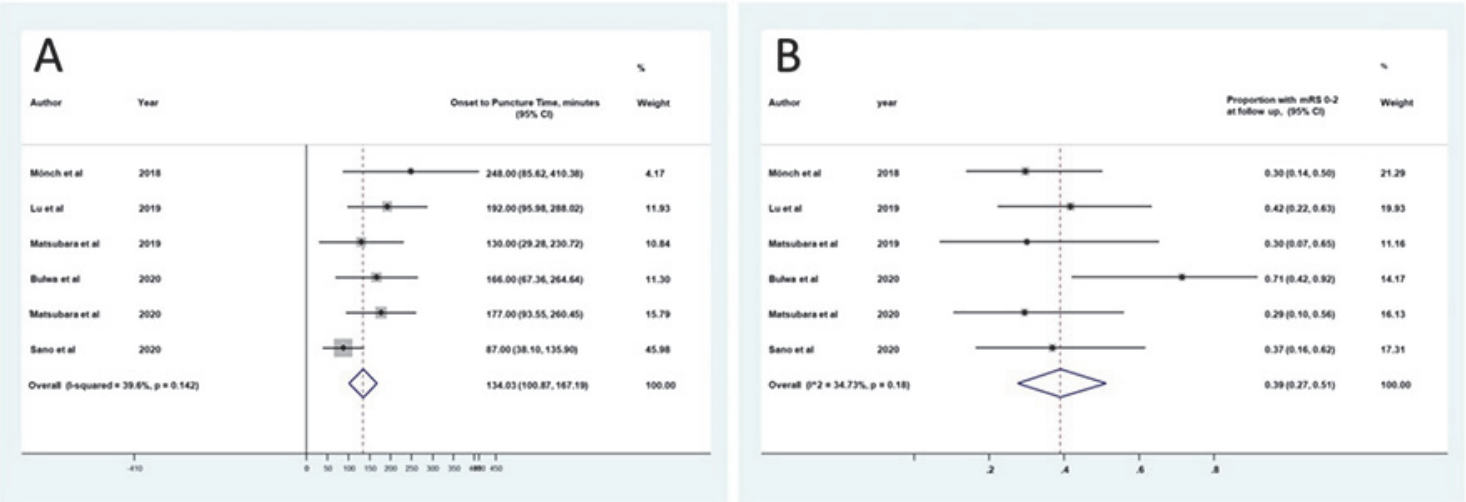